\title{
Reactive vibrational excitation spectroscopy of formic acid in solid argon: Quantum yield for infrared induced trans $\rightarrow$ cis isomerization and solid state effects on the vibrational spectrum
}

\author{
E. M. S. Maçôas ${ }^{a)}$ \\ Department of Chemistry, University of Helsinki, P.O. Box 55 (A.I. Virtasen aukio 1), FIN-00014 Helsinki, \\ Finland and Department of Chemistry (CQC), University of Coimbra, P-3004-535 Coimbra, Portugal \\ L. Khriachtchev, M. Pettersson, b) and J. Juselius \\ Department of Chemistry, University of Helsinki, P.O. Box 55 (A.I. Virtasen aukio 1), \\ FIN-00014 Helsinki, Finland \\ R. Fausto \\ Department of Chemistry (CQC), University of Coimbra, P-3004-535 Coimbra, Portugal \\ M. Räsänen \\ Department of Chemistry, University of Helsinki, P.O. Box 55 (A.I. Virtasen aukio 1), \\ FIN-00014 Helsinki, Finland
}

(Received 4 June 2003; accepted 17 September 2003)

\begin{abstract}
Formic acid molecules are trapped in two predominant local environments (sites) when isolated in an argon matrix at $8 \mathrm{~K}$. Using narrowband tunable infrared (IR) radiation, we performed site-selective excitation of various vibrational modes of the lower-energy trans conformer. For all excited modes, ranging from 7000 to $2950 \mathrm{~cm}^{-1}$, we detected site-selective isomerization to the higher-energy cis form. By measuring the IR absorption of a selected band of the cis conformer as a function of the excitation frequency, the reactive vibrational excitation (RVE) spectra were obtained. The trans $\rightarrow$ cis isomerization quantum yields for the excited modes were determined. Remarkably, very high absolute values were obtained for the quantum yield (up to 40\%) at excitation energies above the reaction barrier. The efficiency of the photoinduced isomerization is essentially independent of the excited vibrational mode in a broad energy interval. Even when the excitation energy was below the reaction barrier, IR-induced rotational isomerization was observed, which indicates tunneling from the vibrationally excited trans conformer to the cis form. Using the RVE spectra, phonon sidebands were detected on the high-frequency side of the zero-phonon-line of the $\mathrm{OH}$ stretching mode of trans-formic acid. These weak and broad bands were not observed in the absorption spectra. Additionally, a relatively narrow band blueshifted by $6 \mathrm{~cm}^{-1}$ from the $\mathrm{OH}$ stretching fundamental mode was assigned to a librational satellite based on simulations using the hindered rotation model for an asymmetric top trapped in an octahedral crystal field. (C) 2003 American Institute of Physics. [DOI: 10.1063/1.1624598]
\end{abstract}

\section{INTRODUCTION}

Infrared (IR)-induced isomerization reactions in cryogenic matrices have been the subject of many studies since the pioneering work on cis- and trans-HONO interconversion by Baldeschwieler and Pimentel. ${ }^{1-3}$ The possibility of mode-selective chemistry and optical control of reaction pathways has been the motivation for many of these studies. Particularly interesting is the conformer-selective photochemistry controlled by narrowband IR irradiation. ${ }^{4}$ Low temperature rare-gas matrices provide an unique environment for selective photochemistry. The low temperatures suppress thermal reactions with significant activation energy, hence enabling stabilization of high energy conformers once they have been produced by photoinduced isomerization.

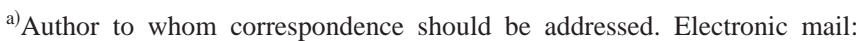
emacoas@qui.uc.pt

b)Present address: Department of Chemistry, University of Jyväskylä, P.O. Box 35, FIN-40014 University of Jyväskylä, Finland.
}

The quantum efficiency of the IR-induced rotational isomerization depends on the probability of energy transfer from the excited vibrational level to the reaction coordinate. For an isolated molecule, the strength of the anharmonic couplings and the density of states are the key factors affecting the relaxation process. ${ }^{5}$ Many unimolecular reactions are often interpreted assuming complete randomization of the excitation energy among all degrees of freedom. This approach is valid if the intramolecular vibrational energy relaxation (IVR) rates are much faster than the reaction rate. Though the IVR dynamics is not simply related to the density of states, complete randomization of the excitation energy is more probable for high energy excitation where the density of states is higher. ${ }^{6}$ On the other hand, if the IVR rates are smaller or comparable with the reaction rate, the reaction efficiency will depend not only on the energy content but also on the initially excited mode. ${ }^{7}$ In condensed phase, the mechanisms for energy relaxation depend additionally on the guest-host interactions. ${ }^{8,9}$ Usually, photoin- 

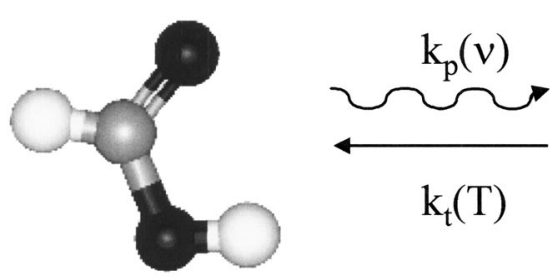

trans- $\mathrm{HCOOH}$

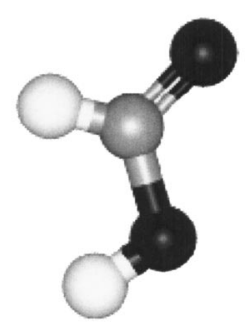

$c i s-\mathrm{HCOOH}$
FIG. 1. Cis and trans formic acid conformers. The trans $\rightarrow$ cis isomerization is induced by vibrational excitation while the inverse cis $\rightarrow$ trans process occurs in dark via tunneling. The tunneling rate is temperature dependent. The cis conformer is higher in energy than the trans form by $1365 \mathrm{~cm}^{-1}$ as estimated experimentally in the gas phase (Ref. 16).

duced rotamerization efficiencies are higher in $\mathrm{Xe}$ than in $\mathrm{Ar}$ matrices, and in solid $\mathrm{Kr}$ the efficiencies are intermediate between those two hosts. ${ }^{10}$ The solid host compensates the energy mismatch between the intramolecular energy levels involved in the energy relaxation by converting the excess internal energy of the guest molecule into lattice phonons. As an extreme case, one may expect the host to favor the relaxation pathway involving lower number of phonons. ${ }^{11}$ For formic acid, the local matrix morphology is expected to influence the dynamics of energy relaxation at low excitation energies (low density of states) due to its effect on the anharmonicity and intermode coupling constants. ${ }^{12}$ Therefore the energy relaxation pathways and consequently photorotamerization efficiencies might depend not only on the rare gas used as host but also on local environment within the same host.

Estimations of quantum yield for narrowband IRinduced rotamerization have been previously reported. , $^{6-15}$ For isomerization of matrix-isolated 2,3-difluoropropene, the quantum yield was shown to depend on excitation energy, on host matrix, and also on the nature of the excited mode. ${ }^{13}$ For $\mathrm{HONO}$, the photoinduced isomerization exhibits mode specific behavior with the efficiency of both cis $\rightarrow$ trans and the trans $\rightarrow$ cis isomerization processes being $\approx 2.5$ times higher for excitation of the $\mathrm{OH}$ stretching fundamental as compared to the first $\mathrm{N}=\mathrm{O}$ stretching overtone, ${ }^{14}$ despite the similar energies of these modes. For $\mathrm{CH}_{2} \mathrm{D}-\mathrm{CH}_{2} \mathrm{D}$ isolated in solid rare gases, the influence of the host and the nature of the excited vibrational mode on the isomerization efficiency were studied by resonant excitation of a number of vibrational states of the gauche and trans conformers. ${ }^{6}$ It was concluded that the conversion efficiencies increase with the excitation energy and the observed deviations from the monotonous behavior were attributed to specific intramolecular interactions controlling the dynamics of the energy relaxation.

Formic acid is one of the simplest molecules with rotational isomerism. It has two stable planar structures, the cis and trans conformers, represented in Fig. 1, the trans form being the conformational ground state. The gas phase energy difference between these two forms was determined as $1365 \pm 30 \mathrm{~cm}^{-1}\left(16 \mathrm{~kJ} \mathrm{~mol}^{-1}\right){ }^{16}$ Extensive spectroscopic studies have been carried out on cis and trans-formic acid isolated in solid Ar, including several isotopologues. ${ }^{12,17-21}$ In an argon matrix, this molecule is mainly isolated in two slightly different local environments as deduced from the IR absorption spectrum, which reveals characteristic site splitting of the bands. ${ }^{12}$

In this work, the IR-induced trans $\rightarrow$ cis isomerization process of formic acid is studied. The quantum yields for the rotamerization induced by selective excitation of various vibrational modes of the trans conformer in the 7000-2950 $\mathrm{cm}^{-1}$ region are determined. As a development of our previous work, ${ }^{22}$ this article emphasizes local-environment effects on the isomerization quantum yields, and the corresponding results on the $\mathrm{OH}$ stretching overtone $(2 \nu \mathrm{OH})$ are added. The solid-state effects on the rotamerization and absorption processes are analyzed. In particular, phonon sidebands and librational satellites of intramolecular vibrational transitions are found using photorotamerization and IR absorption, respectively.

\section{EXPERIMENTAL DETAILS AND METHODOLOGY}

The gaseous samples were prepared by mixing formic acid (KEBO LAB, >99\%), degassed by several freezepump-thaw cycles, with high purity argon (AGA, $99.9999 \%$ ), typically in the 1:2000 proportion. The samples were deposited onto a CsI substrate kept at $15 \mathrm{~K}$ in a closed cycle helium cryostat (APD, DE 202A) and subsequently cooled down to $8 \mathrm{~K}$. Typically $60-70 \mu \mathrm{m}$ thick samples were prepared. The IR absorption spectra $\left(7900-400 \mathrm{~cm}^{-1}\right)$ were measured with a Nicolet SX-60 FTIR spectrometer at 8 $\mathrm{K}$. A liquid-nitrogen-cooled MCT detector and a KBr beamsplitter were used to record the mid-IR spectra, with spectral resolutions from 0.25 to $1.0 \mathrm{~cm}^{-1}$, and a liquid-nitrogencooled InSb detector and a quartz beamsplitter were used for the near-IR spectra, with a spectral resolution of $0.5 \mathrm{~cm}^{-1}$. Typically 100-500 interferograms were co-added.

Tunable pulsed IR radiation provided by an optical parametric oscillator (OPO Sunlite, Continuum, with IR extension) was used to excite vibrationally trans-HCOOH. The pulse duration was $\approx 5 \mathrm{~ns}$ with a spectral linewidth of $\sim 0.1$ $\mathrm{cm}^{-1}$ and a repetition rate of $10 \mathrm{~Hz}$. A Burleigh WA-4500 wavemeter was used to control the OPO radiation frequency, providing an absolute accuracy better than $1 \mathrm{~cm}^{-1}$ for the IR radiation frequency. A mask with a circular hole of $4 \mathrm{~mm}$ in diameter attached on the sample substrate limited the area probed by IR spectroscopy. Since the size of this hole is smaller than both the beam of the spectrometer and the IR pumping beam, the probed area is fully irradiated. The IR radiation energy was measured at the sample position with a pulsed energy meter (Molectron), and it was 0.1-0.6 mJ per pulse for the $2900-7000 \mathrm{~cm}^{-1}$ region. The pumping beam was quasicollinear with the spectrometer beam and the interference filters transmitting in the $2000-500 \mathrm{~cm}^{-1}$ or in the $3300-1100 \mathrm{~cm}^{-1}$ regions attached at the spectrometer detector prevented the pumping radiation from reaching the detector. Another bandpass filter $\left(1500-700 \mathrm{~cm}^{-1}\right)$ placed between the globar source and the sample eliminated globarinduced rotamerization of trans formic acid. These filters limited the spectral window of the IR absorption spectra re- 
corded during pumping to the $1500-700$ or $1500-1100 \mathrm{~cm}^{-1}$ regions.

Photoequilibrium is established under IR pumping of the trans conformer as a result of the interplay between cisformic acid generation and its depletion due to tunneling (see Fig. 1). At the equilibrium the pumping and tunneling rates are equal:

$$
k_{p}(\nu)[\text { trans }]_{\mathrm{eq}}=k_{t}(T)[c i s]_{\mathrm{eq}} .
$$

Hence, the pumping rate $k_{p}(\nu)$ can be determined from the tunneling rate $k_{t}(T)$ and the ratio of the cis and trans photoequilibrium concentrations. For solid argon at $8 \mathrm{~K}, k_{t}(T)$ is $2.3 \times 10^{-3} \mathrm{~s}^{-1}$ and it is not affected by the local environment. ${ }^{23}$ It should be mentioned that the pumping takes place on a time scale (5 ns) which is very short compared with the interval between pulses $(100 \mathrm{~ms})$ and thus essentially an averaged pumping rate is measured. Between the pump pulses the concentration of the cis conformer is practically constant. The concentration of the cis conformer was followed using the integrated absorption of the intense $\mathrm{CO}-\mathrm{COH}$ deformation (CO-COH def.) mode in the 1250$1240 \mathrm{~cm}^{-1}$ region. In the available spectral window to record the IR spectrum during pumping (limited by the filters), there are no bands of trans-formic acid that can be used to follow directly the concentration of this form. Instead, we used the ratio of the absorption cross sections of the $\mathrm{O}-\mathrm{C}=\mathrm{O}$ bending of the trans form ( $80 \mathrm{OCO}$ observed at $\approx 630 \mathrm{~cm}^{-1}$ ) and the $\mathrm{CO}-\mathrm{COH}$ def. band of the cis form to calculate the ratio of the cis and trans photoequilibrium concentrations as follows:

$$
\begin{aligned}
& \Delta A_{T}^{\delta \mathrm{OCO}}=\alpha \Delta A_{C}^{\mathrm{CO}-\mathrm{COHdef}}, \\
& \frac{[\text { cis }]_{\mathrm{eq}}}{[\text { trans }]_{\mathrm{eq}}}=\left(\frac{\Delta A_{T}}{A_{T}^{0}-\Delta A_{T}}\right){ }_{\delta \mathrm{OCO}},
\end{aligned}
$$

where $\Delta A$ is the pumping-induced change of the integrated absorption, $A^{0}$ is the initial (before irradiation) integrated absorption, the subscripts $C$ and $T$ referring to the cis and trans forms, respectively. The value of $\alpha$ was accurately determined from the change of integrated absorptions of the corresponding bands induced by IR pumping in separate experiments without filters.

The pumping rate is proportional to the quantum yield, $\phi(i)$, of the rotamerization process, the absorption cross section of the excited mode of the trans conformer $\left[\sigma_{T}^{i}(\nu)\right.$ in $\left.\mathrm{cm}^{2}\right]$, and the photon intensity of the pumping $\left(I\right.$ in s $\left.{ }^{-1} \mathrm{~cm}^{-2}\right)$ as

$$
k_{p}(\nu)=\phi(i) \sigma_{T}^{i}(\nu) I(\nu),
$$

where $i$ refers to the $i$ th excited vibrational mode. The absorption cross section is obtained using the measured IR absorption at a given excitation frequency, the formic acid concentration and the matrix thickness. Equation (4) is used to calculate the quantum yields once the pumping rates were extracted via Eq. (1).

\section{COMPUTATIONAL MODEL FOR HINDERED ROTATION}

Interaction of molecules with matrix atoms shifts the absorption bands from their gas phase values. The main contribution to the perturbation of the rotational spectra of trapped molecules comes from the electrostatic potential exerted by the lattice atoms, and the coupling of lattice vibrations to the molecular vibration and rotation. The theory of rotation of molecules in condensed phase has been investigated repeatedly. ${ }^{24-30}$ In this work, we use the theoretical description of rotational energy levels of a polyatomic molecule isolated in an octahedral field, developed by Flygare. ${ }^{24}$ This formulation was previously applied for a symmetric top. ${ }^{31}$ In this model, only the electrostatic effects on the rovibrational spectrum are considered and the rotational-translational coupling effects are neglected. Furthermore, quantum effects such as Pauli repulsion due to overlapping electronic wave functions are ignored. This seems to be a good approximation due to the fact that most of the observed vibrations of formic acid isolated in rare-gas matrices are redshifted from the gas-phase values. Although this model is rather simplified, it can provide helpful information for the interpretation of the experimental data with respect to librational motion of the trapped molecule.

A FORTRAN 90 code was written to calculate the Hamiltonian matrix elements in the free rotor basis set according to Flygare's formalism and to diagonalize this matrix. ${ }^{24}$ This FORTRAN 90 code is also used to calculate the dipoletransition moments according to the formulation described in Ref. 31. The program uses as an input three parameters describing the lattice potential $\left(V^{J}, V^{K}, V^{M}\right)$, the gas-phase rotational constants, ${ }^{32,33}$ and the temperature $(T=8 \mathrm{~K})$. The basis set contained 2244 basis functions corresponding to a maximum value of the quantum number $J=15$. The same potential parameters are employed for the fundamental and excited vibrational states. The calculated stick spectrum was displayed using Gaussian functions with a linewidth of 0.6 $\mathrm{cm}^{-1}$. This program was found to reproduce accurately the energy levels of the hindered rotation of linear molecules reported in the literature. ${ }^{29}$ The program also reproduced the symmetric top spectral features obtained in Ref. 31 as a function of temperature for several values of the rotational barrier.

\section{EXPERIMENTAL RESULTS}

The pumping rate for the photoinduced trans $\rightarrow$ cis conversion of formic acid isolated in solid $\operatorname{argon}\left(k_{p}\right)$ was extracted as a function of the excitation frequency as described above. This dependence $\left[k_{p}(\nu)\right]$ is hereafter called a "reactive vibrational excitation (RVE) spectrum," in analogy with Ref. 3. This data is presented in Figs. 2 and 3 for the two major sites observed in an argon matrix. ${ }^{12}$ It has been shown recently that these sites do not interconvert when the matrix is exposed site-selectively to IR radiation. ${ }^{12}$ The molecules with the $\mathrm{OH}$ stretching $(\nu \mathrm{OH})$ absorption at 3548.2 and $3550.5 \mathrm{~cm}^{-1}$ are by definition isolated in site 1 and site 2 , respectively. An equal absorption cross section was assumed for the same vibrational modes of molecules in the two sites. 


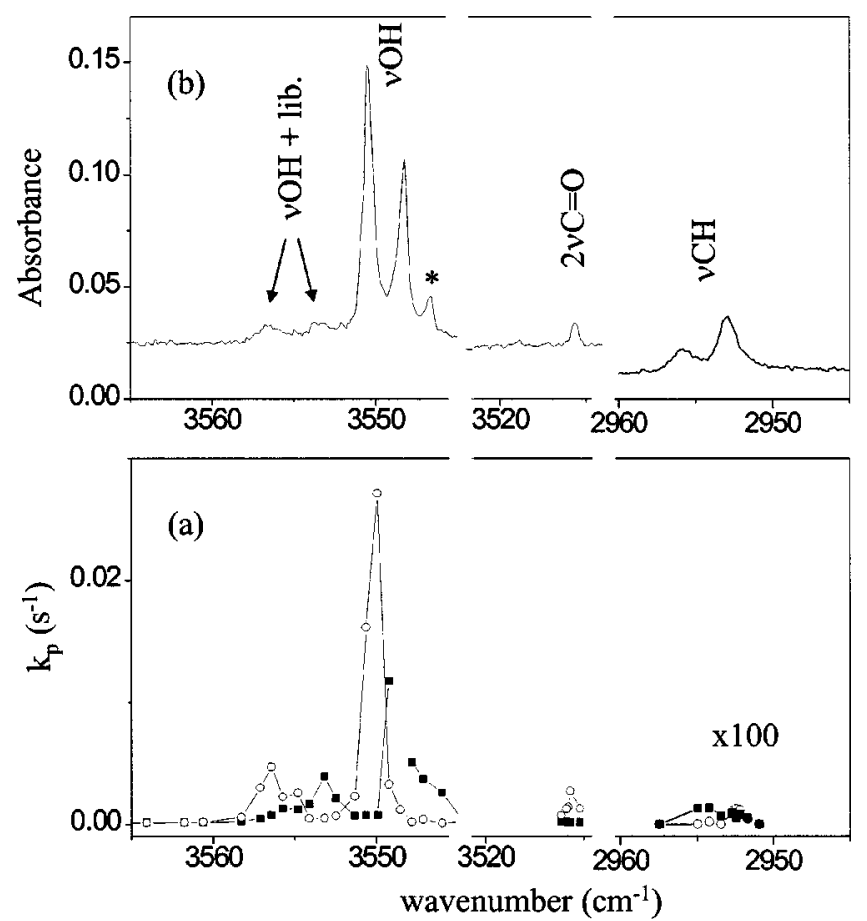

FIG. 2. (a) RVE spectrum for the IR-induced trans $\rightarrow$ cis isomerization of formic acid isolated in solid $\operatorname{Ar}$ at $8 \mathrm{~K}(\boldsymbol{\square}$, site 1 and $\bigcirc$, site 2) and (b) absorption spectrum of trans-formic acid in the $3565-2945 \mathrm{~cm}^{-1}$ region. The RVE spectrum in the $\nu \mathrm{CH}$ region is magnified by a factor of $10^{2}$. The pumping rate value for the excitation of the $\nu \mathrm{OH}$ in site 1 is omitted due to the low accuracy of the measurements. The band marked with (*) was not assigned.

Some support for this approximation was obtained by comparing the relative intensities of various vibrational modes for both sites, which were roughly unaffected by the local environment. This led to the estimation of the site distribution as $62 \%$ of formic acid molecules in site 2 and $38 \%$ in site 1. The absorption spectra are also shown in Figs. 2 and 3 for comparison.

The calculations on formic acid isolated in solid argon lead to an energy difference between the conformers of 1123 $\mathrm{cm}^{-1}\left(13 \mathrm{~kJ} \mathrm{~mol}^{-1}\right)$ and a trans $\rightarrow$ cis energy barrier of 3810 $\mathrm{cm}^{-1}\left(45.6 \mathrm{~kJ} \mathrm{~mol}^{-1}\right){ }^{23}$ Thus, the excitation energies used in this study allowed us to obtain the RVE spectra at energies above the torsional barrier [pumping the $\nu \mathrm{OH}$ overtone $(2 \nu \mathrm{OH})$ at $6930 \mathrm{~cm}^{-1}$ or the combination of the $\nu \mathrm{OH}$ with the $\mathrm{COH}-\mathrm{CO}$ deformation mode $(\nu \mathrm{OH}+\mathrm{COH}-\mathrm{CO}$ def. $)$ at $4650 \mathrm{~cm}^{-1}$ ] and at energies considerably below the barrier [pumping the $\mathrm{CH}$ stretching $(\nu \mathrm{CH})$ at $2950 \mathrm{~cm}^{-1}$ ]. All excited modes in this range were found to be active in promoting the trans $\rightarrow$ cis rotamerization (see Figs. 2 and 3). The RVE spectra were used to determine the quantum yields for the excited vibrations and the results are listed in Table I and plotted as a function of the excitation energy in Fig. 4.

The RVE spectra revealed an interesting and important feature: in addition to the trans $\rightarrow$ cis isomerization induced at exact resonance, the process occurs under pumping at higher energies from the main absorption band. Broad bands blueshifted from the $\nu \mathrm{OH}$ fundamental and overtone modes appear in the RVE spectra (see Fig. 5). A similar behavior is also observed in the case of the combination mode of the
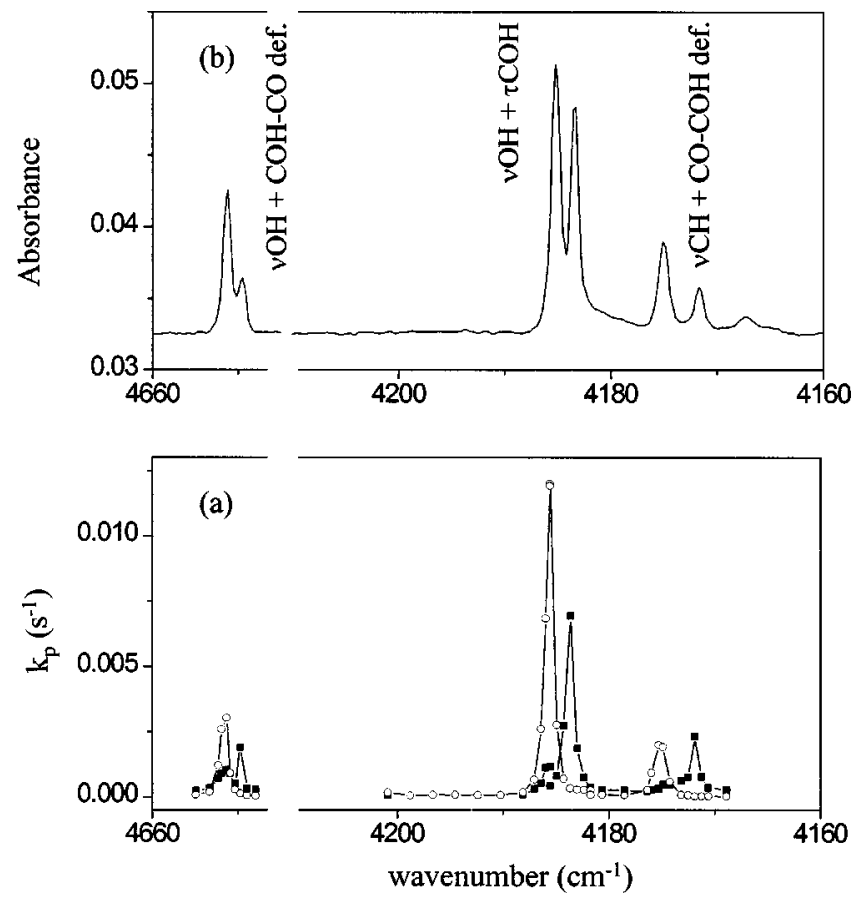

FIG. 3. (a) RVE spectrum for the IR-induced trans $\rightarrow$ cis isomerization of formic acid isolated in solid $\operatorname{Ar}$ at $8 \mathrm{~K}(\boldsymbol{\square}$, site 1 and $O$, site 2) and (b) absorption spectrum of trans-formic in the $4660-4645 \mathrm{~cm}^{-1}$ and $4200-$ $4160 \mathrm{~cm}^{-1}$ regions.

$\nu \mathrm{OH}$ with the $\mathrm{C}-\mathrm{O}$ torsion $\left(\nu \mathrm{OH}+\tau \mathrm{COH}\right.$ at $\left.4184 \mathrm{~cm}^{-1}\right)$. The broadness and low intensities of these sidebands prevent their direct observation in the IR absorption spectra.

As seen in Figs. 2 and 3, the relative peak intensities differ in the RVE and IR absorption spectra. The most significant difference is observed for the $\mathrm{CH}$ stretching mode $(\nu \mathrm{CH})$ whose pumping rate is at least 2 orders of magnitude lower than for the $\nu \mathrm{OH}$ fundamental or the carbonyl stretching overtone $(2 \nu \mathrm{C}=\mathrm{O})$. A reliable difference is also observed in the $\nu \mathrm{OH}$ region for site 2 when comparing the relative intensities of the $\nu \mathrm{OH}\left(3550.5 \mathrm{~cm}^{-1}\right)$ band and the narrow blueshifted satellite band at $3556.7 \mathrm{~cm}^{-1}$ (see Fig. 2). For site 2, the IR absorption spectrum of this region is shown

TABLE I. Quantum yield for the IR-induced trans $\rightarrow$ cis rotamerization in formic acid. ${ }^{\mathrm{a}}$

\begin{tabular}{|c|c|c|c|c|}
\hline \multirow[b]{2}{*}{ Excited mode } & \multicolumn{4}{|c|}{ Quantum yield $(\phi)$} \\
\hline & Site 1 & & Site 2 & \\
\hline $2 \nu \mathrm{OH}$ & 0.17 & $(6930.3)$ & 0.07 & $(6934.8)$ \\
\hline$\nu \mathrm{OH}+\mathrm{COH}-\mathrm{CO}$ def. & 0.15 & $(4649.6)$ & 0.21 & (4651.4) \\
\hline$\nu \mathrm{OH}+\tau \mathrm{COH}$ & 0.21 & (4183.6) & 0.41 & (4185.2) \\
\hline$\nu \mathrm{CH}+\mathrm{CO}-\mathrm{COH}$ def. & 0.25 & (4171.8) & 0.20 & $(4175.1)$ \\
\hline$\nu \mathrm{OH}+$ lib. & 0.050 & $(3553.7)$ & 0.12 & (3556.7) \\
\hline$\nu \mathrm{OH}$ & $\mathrm{b}$ & $(3548.2)$ & 0.039 & $(3550.5)$ \\
\hline$*$ & 0.024 & $(3546.7)$ & $\ldots$ & $\ldots$ \\
\hline $2 \nu \mathrm{C}=\mathrm{O}$ & $\ldots$ & $(3519.0)$ & 0.051 & (3515.7) \\
\hline$\nu \mathrm{CH}$ & $1.4 \times 10^{-4}$ & $(2956.1)$ & $8.4 \times 10^{-5}$ & (2953.1) \\
\hline
\end{tabular}

${ }^{a}$ The position of the absorption band of each site is given in parentheses $\left(\mathrm{cm}^{-1}\right)$. Symbols: $\nu$, stretching; def., deformation; $\tau$, torsion; lib., libration.

${ }^{\mathrm{b}}$ This value is omitted due to low accuracy of the measurements determined by the very low concentration of trans- $\mathrm{HCOOH}$ in site 1 in the photoequilibrium. The absorption band marked with (*) is not assigned. 


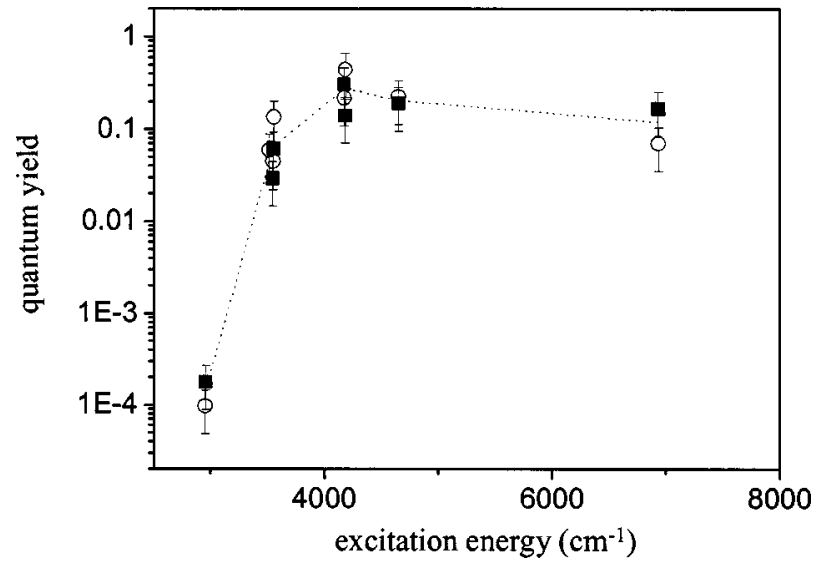

FIG. 4. Quantum yield (in logarithmic scale) for IR-induced trans $\rightarrow$ cis isomerization of $\mathrm{HCOOH}$ isolated in $\mathrm{Ar}$ at $8 \mathrm{~K}$ as a function of the excitation energy ( $\mathbf{\square}$, site 1 and $\bigcirc$, site 2$)$. The points correspond to the following vibrational modes: $\sim 6930 \mathrm{~cm}^{-1}: 2 \nu \mathrm{OH} ; \sim 4650 \mathrm{~cm}^{-1}: \nu \mathrm{OH}+\mathrm{COH}-\mathrm{CO}$ def.; $\sim 4185 \mathrm{~cm}^{-1}: \nu \mathrm{OH}+\tau \mathrm{COH} ; \sim 4175 \mathrm{~cm}^{-1}: \nu \mathrm{CH}+\mathrm{CO}-\mathrm{COH}$ def.; $\sim 3550 \mathrm{~cm}^{-1}: \nu \mathrm{OH} ; \sim 3515 \mathrm{~cm}^{-1}: 2 \nu \mathrm{C}=\mathrm{O} ; \sim 2955 \mathrm{~cm}^{-1}: \nu \mathrm{CH}$. The estimated energy barrier for the reaction is $3810 \mathrm{~cm}^{-1}$. A dotted line guides the eye.

in detail in Fig. 6. The narrow blueshifted satellite band is here assigned to a combination between the $\nu \mathrm{OH}$ mode and the librational motion of the molecule $(\nu \mathrm{OH}+$ lib.). As shown in Fig. 6, a similar blueshifted librational satellite band was observed for $\mathrm{DCOOH}$. This assignment is supported by simulations of hindered rotation of the trapped molecule in the matrix cage to be discussed below.

\section{DISCUSSION}

\section{A. Quantum yield for the trans $\rightarrow$ cis isomerization}

Four combination modes of trans-formic acid with energies above the isomerization barrier were pumped siteselectively: $2 \nu \mathrm{OH}\left(6932 \mathrm{~cm}^{-1}\right), \nu \mathrm{OH}+\mathrm{COH}-\mathrm{CO}$ def. (4650 $\left.\mathrm{cm}^{-1}\right), \nu \mathrm{OH}+\tau \mathrm{COH}\left(4184 \mathrm{~cm}^{-1}\right)$, and $\nu \mathrm{CH}+\mathrm{CO}-\mathrm{COH}$ def. $\left(4173 \mathrm{~cm}^{-1}\right) .{ }^{12}$ At these excitation energies, the isomerization quantum yield is $\sim 20 \%$ (see Table I). The efficiency of the process is rather independent of the excited modes and no major differences between the trapping sites are observed either (see Fig. 4). Interestingly, the quantum yield values determined for this relatively small molecule are very large when compared to the values reported for $\mathrm{CH}_{2} \mathrm{FC}(\mathrm{F})=\mathrm{CH}_{2}\left(10^{-7}-10^{-4}\right) .{ }^{13}$ Similarly high quantum yields were previously estimated for the photoisomerization process in the HONO molecule by excitation of the $\nu \mathrm{OH} .{ }^{14}$ The high quantum yields imply that the time scale for the trans $\rightarrow$ cis isomerization is shorter than the time scale for vibrational relaxation within the trans well. The latter is dictated by the rate of energy transfer to the lattice which, in turn, depends on the energy gaps between the intramolecular vibrational levels. For small molecules, such as formic acid, there may exist large energy gaps acting as bottlenecks for relaxation.

For pumping above the isomerization barrier, there are some fluctuations of the quantum yield values to be noticed in Table I. For example, in site 2, quantum yields for the $2 \nu \mathrm{OH}$ and $\nu \mathrm{OH}+\tau \mathrm{COH}$ modes differ by a factor of 6 . The
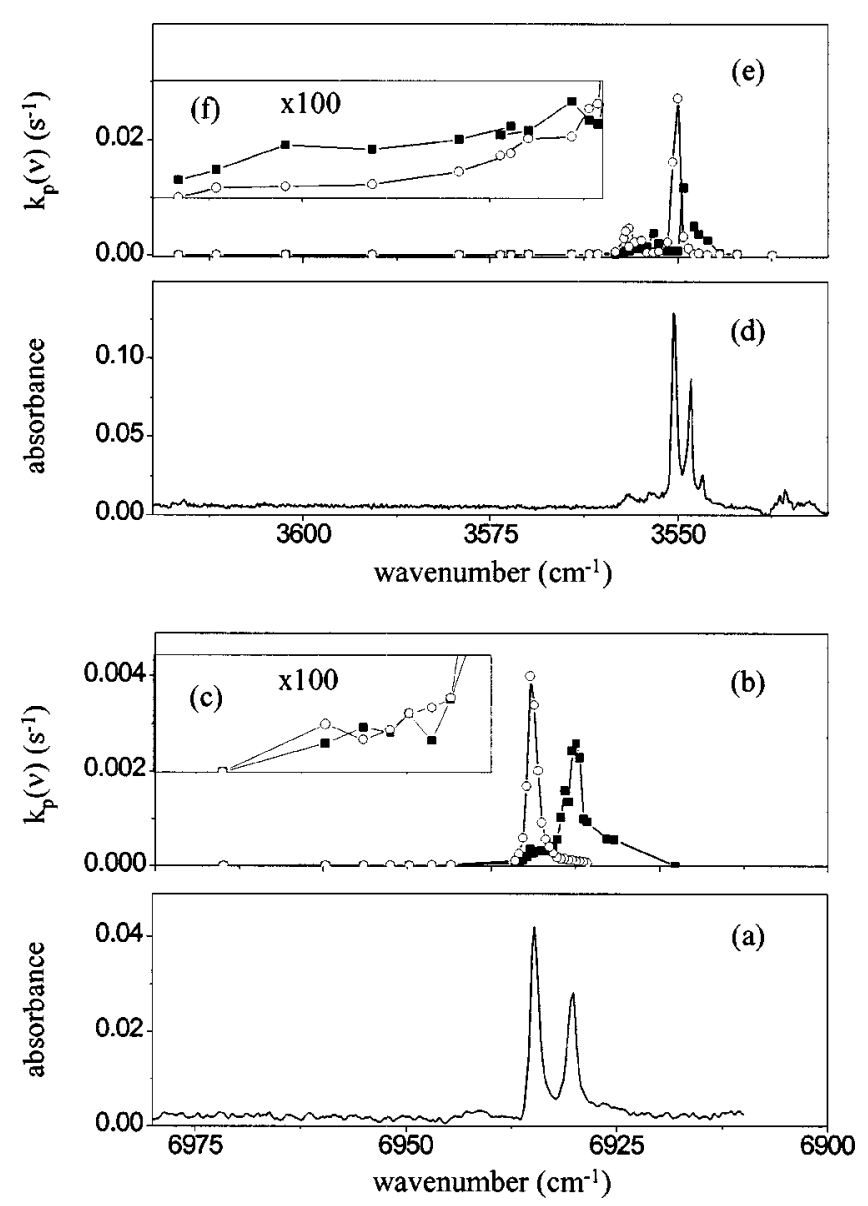

FIG. 5. Spectra of formic acid in the $2 \nu \mathrm{OH}(\mathrm{a}-\mathrm{c})$ and $\nu \mathrm{OH}(\mathrm{d}-\mathrm{f})$ region showing the presence of the phonon sideband in the RVE spectra $(\mathbf{\square}$, site 1 and $\bigcirc$, site 2): (a) and (d) IR-absorption spectra. (b) and (e) RVE spectra; (c) and (f) expansion of the RVE spectra. The absorption maxima should not be compared between these two spectral regions because different matrices were used to collect these spectra.

quantum yield for the $\nu \mathrm{OH}+\tau \mathrm{COH}$ mode differs by a factor of 2 depending on the site (see Table I). These results could be considered as some limited evidence of site- and modespecific effects. The observed deviations from the averaged value are rather small compared, for example, with the variations of quantum yield reported for deuterated ethane (order of magnitude). ${ }^{6}$ In our experiments, the quantum yield extracted for $2 \nu \mathrm{OH}$ has somewhat larger uncertainty (compared with the other modes) due to experimental reasons.

The quantum yield was also estimated for four vibrations below the predicted torsional barrier: combination of $\nu \mathrm{OH}$ with the librational motion of the molecule $(\nu \mathrm{OH}+$ lib., 3555 $\left.\mathrm{cm}^{-1}\right), \nu \mathrm{OH}\left(3549 \mathrm{~cm}^{-1}\right), 2 \nu \mathrm{C}=\mathrm{O}\left(3516 \mathrm{~cm}^{-1}\right)$, and $\nu \mathrm{CH}$ $\left(2955 \mathrm{~cm}^{-1}\right)$. The value obtained for the $\nu \mathrm{OH}$ mode in site 2 is lower by a factor of 3 than that for its librational satellite (see Table I). In a study on librational motion of the hydroxyl ion isolated in alkali halide crystals, it was suggested that the excited stretching and stretching-libration combination states should have different decay mechanisms. ${ }^{34}$ The enhanced efficiency of the combination mode may be due to the stronger coupling between this mode and the reaction coordinate. For $\mathrm{HONO}$, it was previously suggested that the presence of an- 


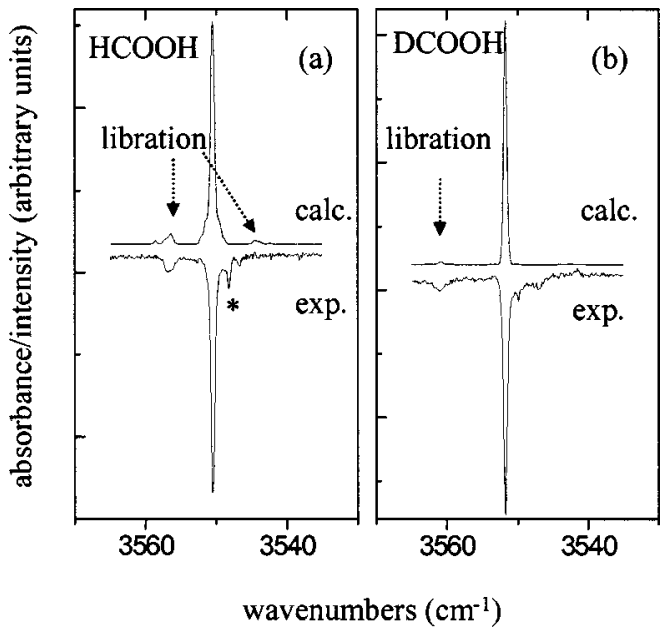

FIG. 6. IR absorption bands observed in the $\nu \mathrm{OH}$ region of $\mathrm{HCOOH}$ and $\mathrm{DCOOH}$ isolated in site 2 in an Ar matrix at $8 \mathrm{~K}$ (downwards) and simulated spectra on the basis of the hindered rotation of the molecule in the matrix (upwards). The experimental spectra are difference spectra showing the result of the site-selective pumping of molecules in site 2 . The band marked with (*) belongs to trans- $\mathrm{HCOOH}$ in site 1 and it is seen here due to the limited site-selectivity caused by the overlap of the absorption bands at the irradiation wavelength. For the simulated spectra, the calculated transitions on the basis of the hindered rotation model (Ref. 24) were plotted with Gaussian bands $\left(\mathrm{FWHM}=0.6 \mathrm{~cm}^{-1}\right)$.

gular momentum enhances the energy transfer to the torsional mode. $^{35}$

The energy dependence of the quantum yield for excitation below the energy barrier for the trans $\rightarrow$ cis conversion is attributed to the tunneling between the trans and cis potential wells. ${ }^{23}$ At these excitation energies, the quantum yield is limited by the tunneling probability between the potential wells. On the other hand, there are no such limitations for the quantum yield when the molecule is pumped with energy above the torsional barrier. The energy relaxation mechanism that channels the excitation energy into the reaction coordinate is clearly very complex, and its thorough theoretical investigation is highly desirable.

\section{B. Phonon-sideband excitation}

The RVE spectra reveal broad bands at higher energies from intramolecular vibrations (see Fig. 5). Photoinduced processes activated far from obvious absorption bands have been earlier assigned to excitation of two kinds of weak combinations: librational modes of the isolated molecule or lattice phonons with intramolecular vibrations. ${ }^{36,37}$ In this work, the broad satellite bands in the RVE spectrum are characteristic of phonon sidebands of intramolecular vibrational transitions observed in pure molecular solids, ${ }^{38}$ the zerophonon-line (ZPL) corresponding to the intramolecular vibration. These phonon sidebands originate from the simultaneous excitation of an intramolecular vibration and a lattice phonon by one photon. Intense phonon sidebands are commonly observed for electronic transitions ${ }^{39,40}$ when the most probable vertical transition involves a certain number of lattice phonons. ${ }^{41}$ In most of the cases, the vibrational spectra of matrix-isolated species consist mainly of sharp ZPLs showing little indications of phonon sidebands. This difference is due to weaker vibration-phonon coupling compared with electron-phonon coupling.

The calculated one-phonon density of states of pure solid argon is about $70 \mathrm{~cm}^{-1}$ broad, ${ }^{39,42,43}$ which is in agreement with the $\approx 65 \mathrm{~cm}^{-1}$ wide band observed in the RVE spectrum above the $\nu \mathrm{OH}$ band $\left(3549 \mathrm{~cm}^{-1}\right)$. Pumping at energies below the ZPL is inefficient in promoting isomerization, which is due to the low probability of the anti-Stokes process (when an existing phonon contributes to the vibrational transition) at the matrix temperatures. The ratio of the intensity of the phonon sideband to the ZPL is a measure of the coupling of the involved vibration with the lattice phonons. ${ }^{39}$ In the RVE spectra, the measured ratio of the pumping rates for these two bands is $\sim 0.08$. The actual ratio of the absorption bands may differ from this value if the quantum yield differs between the ZPL and the phonon sideband. As shown in Fig. 5, for the $2 \nu \mathrm{OH}$ the apparently nonresonant excitation was detected up to $40 \mathrm{~cm}^{-1}$ from the ZPL. Our sensitivity to probe the phonon sideband of this mode is limited due to the fact that the ZPL is more than one order of magnitude weaker than the ZPL of the fundamental vibration. Nevertheless, some estimation of the coupling efficiency of the $2 \nu \mathrm{OH}$ mode can be made comparing the ratio of the absolute intensities of the ZPL and that of the phonon sideband at $\sim 20$ $\mathrm{cm}^{-1}$ from the ZPL for the $\nu \mathrm{OH}$ fundamental and overtone modes. Within our accuracy, this gives similar coupling efficiency for both modes.

The RVE spectrum appears to be useful to probe phonon sidebands that are in most cases not observed in the IR absorption spectrum. The increase of sensitivity to the weak and broad spectral features in the RVE method as compared with the IR absorption is explained by the essential absence of noisy background. Notice that the signals in the RVE spectra come from the efficiency of a given excited vibration to induce the isomerization reaction. The pumping rate is determined indirectly from the absorption of a certain vibrational mode of the reaction product (cis- $\mathrm{HCOOH}$ here), which was chosen due to its large absorption cross section in order to maximize sensitivity. In other words, this advantage is analogous to the situation with the high sensitivity of luminescence spectroscopy as compared with absorption spectroscopy. As shown above, a significant part of the oscillator strength can be carried by the phonon sideband. This possibility should be kept in mind when making accurate determinations of the band intensities in solid matrices. ${ }^{31}$ In addition, while studying processes promoted by IR light one should be aware that due to phonon sidebands the processes can be induced over a broad spectral range in an apparently nonresonant way.

\section{Librational motion}

The IR absorption spectrum in the $\nu \mathrm{OH}$ spectral region of $\mathrm{HCOOH}$ and $\mathrm{DCOOH}$ isolated in solid Ar reveals the presence of narrow bands blueshifted by 6 and $9 \mathrm{~cm}^{-1}$ from the fundamental absorptions, respectively. In this section, we will discuss the assignment of these bands to the combination of the librational and vibrational motions of the molecule. 
For $\mathrm{HCOOH}$, site 2 exhibits one weak blueshifted satellite at $3556.7 \mathrm{~cm}^{-1}$ in addition to the main $\nu \mathrm{OH}$ fundamental band at $3550.5 \mathrm{~cm}^{-1}$ (see Fig. 6). ${ }^{12} \mathrm{HCOOH}$ in site 1 shows one main band due to the $\nu \mathrm{OH}$ fundamental mode at 3548.2 $\mathrm{cm}^{-1}$ and two weak satellite bands at 3553.7 and 3546.7 $\mathrm{cm}^{-1} \cdot{ }^{12}$ Simply by summing the fundamental transitions, the combination modes that could explain the observed satellites are the $\nu \mathrm{CH}$ plus $\mathrm{OCO}$ deformation $(\nu \mathrm{CH}+\delta \mathrm{OCO} \sim 3583$ $\left.\mathrm{cm}^{-1}\right)$ and the $\nu \mathrm{CH}$ plus $\mathrm{COH}$ torsion $(\nu \mathrm{CH}+\tau \mathrm{COH} \sim 3590$ $\mathrm{cm}^{-1}$ ), which are $20-40 \mathrm{~cm}^{-1}$ higher than the observed bands. For DCOOH, site 2 exhibits one weak blueshifted satellite at $3560.9 \mathrm{~cm}^{-1}$ in addition to the $\nu \mathrm{OH}$ main band at $3551.7 \mathrm{~cm}^{-1}$ (see Fig. 6). ${ }^{12}$ Site 1 of DCOOH shows two weak satellite bands at 3562.5 and $3547.2 \mathrm{~cm}^{-1}$ and one main band assigned to $\nu \mathrm{OH}$ at $3549.9 \mathrm{~cm}^{-1}$. $^{12}$ The observation of a similar absorption pattern in the $\nu \mathrm{OH}$ spectral region of both isotopologues shows that the weak satellites observed cannot originate from combination modes involving the $\nu \mathrm{CH}$ vibration. No other second order combinations or overtones without involving the $\mathrm{C}-\mathrm{H}$ bond are expected in this region.

In a recent paper, the rotationally resolved $\nu \mathrm{OH}$ and $\nu \mathrm{CH}$ spectral regions of $\mathrm{HCOOH}$ and $\mathrm{DCOOH}$ monomers were analyzed in liquid He droplets. ${ }^{44}$ In that study, the accidental resonant mixing between the $\nu \mathrm{OH}$ and the $\nu \mathrm{CH}+8 \mathrm{OCO}$ and $\nu \mathrm{CH}+\tau \mathrm{COH}$ combination modes was shown to give rise to a triplet at 3571,3569 , and $3566 \mathrm{~cm}^{-1}$. In He droplets, the $\nu \mathrm{OH}$ fundamental couples via Fermi resonance with the $\nu \mathrm{CH}$ $+8 \mathrm{OCO}$ combination mode that, in turn, interacts with the $\nu \mathrm{CH}+\tau \mathrm{COH}$ mode via Coriolis coupling. ${ }^{44}$ The assignment made for the observed bands in He droplets was supported by the absence of the same spectral pattern in the $\nu \mathrm{OH}$ region of the $\mathrm{DCOOH}$ isotopologue. This indicates different origins for the weak blueshifted bands observed in Ar matrix and in He droplets.

The coupling of the $\nu \mathrm{OH}$ mode with the librational motion of the molecule can explain the observed spectrum for the two isotopologues in the $\nu \mathrm{OH}$ region. Several small molecules have been shown to exhibit rotation in rare gas matrices. ${ }^{8,9,31,45-47}$ Hindered rotation of a molecule in the matrix cage gives rise to librational motion.

In the simulations, the three components of the rotational barrier $\left[V^{J}, V^{K}, V^{M}\right]$ were taken as free parameters to fit the calculated rovibrational spectrum to the observed spectrum in the $\nu \mathrm{OH}$ region for both $\mathrm{HCOOH}$ and $\mathrm{DCOOH}$. As expected, for $V^{J}=V^{K}=V^{M}=0 \mathrm{~cm}^{-1}$ the spectra show the characteristic pattern of free rotation. When the rotational barriers are large enough the hindered rotation gives rise to two satellite bands equally shifted to higher and lower energies from the main band. The shift of the satellite bands from the main band increases and their intensity decrease as the barrier increases. The blueshifted satellite band corresponds to the sum combination while the redshifted band corresponds to the difference combination. The expected lower population of the excited librational levels at the low working temperature $(8 \mathrm{~K})$ determines the weaker intensity of the redshifted satellites when compared with the blueshifted ones.

The position of the librational satellite was found to be essentially dependent on the $V^{J}$ value and rather insensitive to the $V^{K}$ and $V^{M}$ values. Reasonably good fits to the experimental values were obtained with $V^{J}=11 \mathrm{~cm}^{-1}$ for $\mathrm{HCOOH}$ and $V^{J}=18 \mathrm{~cm}^{-1}$ for DCOOH, irrespectively of the value of $V^{K}$ and $V^{M}$, which were varied independently from 0 to 100 $\mathrm{cm}^{-1}$. As an illustration, in Fig. 6 the spectra obtained with the optimized $V^{J}$ values for both isotopologues and $V^{K}$ $=V^{M}=0 \mathrm{~cm}^{-1}$ is shown. Some isotopic effect is expected due to the different hexadecapole moments of the two isotopologues arising from the different position of the center of mass for the $\mathrm{O}-\mathrm{H}$ and $\mathrm{O}-\mathrm{D}$ forms. As shown in Fig. 6, the hindered rotation model reproduces the position and relative intensity of the blueshifted satellite of the $\nu \mathrm{OH}$ observed for both $\mathrm{HCOOH}$ and $\mathrm{DCOOH}$, thus giving confidence to the assignment. The redshifted bands were not experimentally observed, which is also in agreement with the simulations that give a very low intensity for these bands. Though this qualitative explanation of the observed satellites is based on a simple application of Flygare's model, the main features of the experimental spectra are well reproduced. A more detailed theoretical analysis is outside the scope of the present work.

In addition to the blueshifted satellite, for site 1 of $\mathrm{HCOOH}$ and $\mathrm{DCOOH}$ it is also possible to observe a band that is about $2 \mathrm{~cm}^{-1}$ redshifted from the pure $\nu \mathrm{OH}$ fundamental band (see Fig. 2). This band cannot be associated with a librational band due to its small shift from the fundamental and its higher intensity when compared with the blueshifted librational satellite. Currently we have no explanation for this band.

\section{CONCLUDING REMARKS}

The trans $\rightarrow$ cis rotamerization of formic acid in solid Ar was induced site- and mode-selectively by using narrowband IR irradiation in the 7000-2950 $\mathrm{cm}^{-1}$ region. All excited modes in this region were active in promoting the isomerization process. Remarkable is the high absolute value of the quantum yield (up to $40 \%$ ) estimated for this isomerization process. While the details of the isomerization mechanism are currently unknown this very high efficiency should be of interest for theoretical modeling. The energy relaxation is rather independent of both the nature of excited mode and the trapping site. Nevertheless, the observed deviations from the average quantum yield value of $20 \%$ for excitation energies above the rotamerization barrier could be an indication of some specific mode and local environment effects. This interpretation has to be considered tentative due to the limited accuracy of the present experiments. Tunneling is an important mechanism involved in the rotamerization of formic acid essentially evidenced by the trans $\rightarrow$ cis isomerization induced by excitation of the $\nu \mathrm{CH}$ mode. Excitation of this mode introduces an energy in the system which is considerably below the energy barrier.

The RVE spectrum reveals the solid state effects on the vibrational spectroscopy of formic acid isolated in an Ar matrix. Broad RVE bands blueshifted from the intramolecular vibrations were assigned to phonon sidebands. In general, such weak and broad bands are not observed in the IR absorption spectra. Additionally, narrower bands blueshifted 
from the pure $\nu \mathrm{OH}$ band observed both in the RVE and IR absorption spectra of $\mathrm{HCOOH}$ were assigned to librational satellites.

\section{ACKNOWLEDGMENTS}

The Academy of Finland is thanked for financial support. E.M.S.M. and R.F. acknowledge the Portuguese Foundation for Science and Technology (Ph.D. Grant No. SFRH/ BD/4863/2001 and Project No. POCTI/43366/QUI/2001).

${ }^{1}$ J. D. Baldeschwieler and G. C. Pimentel, J. Chem. Phys. 33, 1008 (1960).

${ }^{2}$ H. Frei and G. C. Pimentel, Annu. Rev. Phys. Chem. 36, 491 (1985).

${ }^{3} \mathrm{H}$. Frei and G. C. Pimentel, in Chemistry and Physics of Matrix-Isolated Species, edited by L. Andrews and M. Moskovits (Elsevier Science, New York, 1989).

${ }^{4}$ L. Khriachtchev, E. Maçôas, M. Pettersson, and M. Räsänen, J. Am. Chem. Soc. 124, 10994 (2002).

${ }^{5}$ J. Nesbitt and R. W. Field, J. Phys. Chem. 100, 12735 (1996).

${ }^{6}$ P. Roubin, S. Varin, P. Verlaque, S. Coussan, J.-M. Berset, J.-M. Ortéga, A. Peremans, and W.-Q. Zheng, J. Chem. Phys. 107, 7800 (1997).

${ }^{7}$ J. Jortner and R. D. Levine, in Advances in Chemical Physics, edited by J. Jortner, R. D. Levine, and S. A. Rice (Wiley-Interscience, New York, 1981), Vol. 47.

${ }^{8}$ V. E. Bondybey, M. Räsänen, and A. Lammers, Annu. Rep. Prog. Chem., Sect. C: Phys. Chem. 95, 331 (1999).

${ }^{9}$ F. Legay, in Chemical and Biochemical Applications of Lasers, edited by C. B. Moore (Academic, New York, 1977).

${ }^{10}$ M. Räsänen, H. Kunttu, and J. Murto, Laser Chem. 9, 123 (1988).

${ }^{11}$ V. E. Bondybey, Annu. Rev. Phys. Chem. 35, 591 (1984).

${ }^{12}$ E. M. S. Maçôas, J. Lundell, M. Pettersson, L. Khriachtchev, R. Fausto, and M. Räsänen, J. Mol. Spectrosc. 219, 70 (2003).

${ }^{13}$ A. K. Knudsen and G. C. Pimentel, J. Phys. Chem. 95, 2823 (1991)

${ }^{14}$ L. Khriachtchev, J. Lundell, E. Isoniemi, and M. Räsänen, J. Chem. Phys. 113, 4265 (2000).

${ }^{15}$ W. F. Hoffman III and J. S. Shirk, Chem. Phys. 78, 331 (1983).

${ }^{16}$ W. M. Hocking, Z. Naturforsch. A 31A, 1113 (1976).

${ }^{17}$ M. Pettersson, J. Lundell, L. Khriachtchev, and M. Räsänen, J. Am. Chem. Soc. 119, 11715 (1997).

${ }^{18}$ T. Miyazawa and K. S. Pitzer, J. Chem. Phys. 30, 1076 (1959).

${ }^{19}$ R. L. Redington, J. Mol. Spectrosc. 65, 171 (1977).

${ }^{20}$ D. O. Henderson, Doctoral thesis, Texas Tech. University, 1987.
${ }^{21}$ F. Madeja, P. Markwick, M. Havenith, K. Nauta, and R. E. Miller, J. Chem. Phys. 116, 2870 (2002).

${ }^{22}$ M. Pettersson, E. M. S. Maçôas, J. Lundell, L. Khriachtchev, R. Fausto, and M. Räsänen, J. Chem. Phys. 117, 9095 (2002).

${ }^{23}$ M. Pettersson, E. M. S. Maçôas, L. Khriachtchev, R. Fausto, and M. Räsänen, J. Am. Chem. Soc. 125, 4058 (2003).

${ }^{24}$ H. Flygare, J. Chem. Phys. 39, 2263 (1963).

${ }^{25}$ A. Cabana, G. B. Savitzky, and D. F. Hornig, J. Chem. Phys. 39, 2942 (1963)

${ }^{26}$ V. Narayanamurti, Phys. Rev. Lett. 13, 693 (1964).

${ }^{27}$ B. Wedding and M. V. Klein, Bull. Am. Phys. Soc. 11, 228 (1966).

${ }^{28}$ A. F. Devonshire, Proc. R. Soc. London, Ser. A 153, 601 (1936).

${ }^{29}$ H. F. King and D. F. Hornig, J. Chem. Phys. 44, 4520 (1966).

${ }^{30}$ P. Sauer, Z. Phys. 194, 360 (1966).

${ }^{31}$ V. A. Apkarian and E. Weitz, J. Chem. Phys. 76, 5796 (1982).

${ }^{32}$ J. Vander Auwera, J. Mol. Spectrosc. 155, 136 (1992).

${ }^{33}$ K. L. Goh, P. P. Ong, T. L. Tan, W. F. Wan, and H. H. Teo, J. Mol. Spectrosc. 190, 125 (1998).

${ }^{34}$ M. V. Klein, B. Wedding, and M. A. Levine, Phys. Rev. 180, 902 (1969).

${ }^{35}$ P. M. Agrawal, D. L. Thompson, and L. M. Raff, J. Chem. Phys. 102, 7000 (1995).

${ }^{36}$ S. Fei, G. S. Yu, H. W. Li, and H. L. Strauss, J. Chem. Phys. 104, 6398 (1996).

${ }^{37}$ W. P. Ambrose and A. J. Sievers, J. Opt. Soc. Am. B 9, 753 (1992).

${ }^{38}$ A. P. Brodyanski, S. A. Medvedev, M. Vetter, J. Kreutz, and H. J. Jodl, Phys. Rev. B 66, 104301 (2002).

${ }^{39}$ V. E. Bondybey and C. Fletcher, J. Chem. Phys. 64, 3615 (1976).

${ }^{40}$ M. Pettersson and J. Nieminen, Chem. Phys. Lett. 283, 1 (1998).

${ }^{41}$ V. E. Bondybey, in Chemistry and Physics of Matrix-Isolated Species, edited by L. Andrews and M. Moskovits (Elsevier Science, Amesterdam, 1989).

${ }^{42}$ Rare Gas Solids, edited by M. L. Klein and J. A. Venables (Academic, London, 1976), Vol. 1.

${ }^{43}$ H. J. Jodl, in Chemistry and Physics of Matrix-Isolated Species, edited by L. Andrews and M. Moskovits (Elsevier Science, Amesterdam, 1989).

${ }^{44}$ F. Madeja, P. Markwick, and M. Havenith, J. Chem. Phys. 116, 2870 (2002).

${ }^{45}$ L. H. Jones, S. A. Ekberg, and B. I. Swanson, J. Chem. Phys. 85, 3203 (1986).

${ }^{46}$ R. M. Bentwood, A. J. Barnes, and W. J. Orville-Thomas, J. Mol. Spectrosc. 84, 391 (1980).

${ }^{47}$ E. Isoniemi, M. Pettersson, L. Khriachtchev, J. Lundell, and M. Räsänen, J. Phys. Chem. A 103, 679 (1999). 
The Journal of Chemical Physics is copyrighted by the American Institute of Physics (AIP). Redistribution of journal material is subject to the AIP online journal license and/or AIP copyright. For more information, see http:/ojps.aip.org/jcpo/jcpcr/jsp Copyright of Journal of Chemical Physics is the property of American Institute of Physics and its content may not be copied or emailed to multiple sites or posted to a listserv without the copyright holder's express written permission. However, users may print, download, or email articles for individual use. 
The Journal of Chemical Physics is copyrighted by the American Institute of Physics (AIP). Redistribution of journal material is subject to the AIP online journal license and/or AIP copyright. For more information, see http://ojps.aip.org/jcpo/jcper/jsp 